\title{
A NOVEL TECHNIQUE FOR PRECISION GEOMETRIC CORRECTION OF JITTER DISTORTION FOR THE EUROPA IMAGING SYSTEM AND OTHER ROLLING-SHUTTER CAMERAS
}

\author{
R. L. Kirk ${ }^{\mathrm{a}}$, M. Shepherd ${ }^{\mathrm{a}}$, and S. C. Sides ${ }^{\mathrm{a}}$

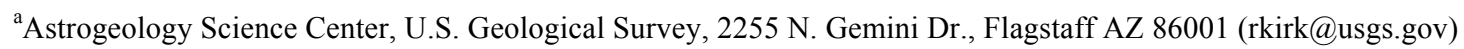

\author{
Commission III, ICWG III/II
}

KEY WORDS: Europa, photogrammetry, jitter, geometric correction, active-pixel sensors

\begin{abstract}
:
We use simulated images to demonstrate a novel technique for mitigating geometric distortions caused by platform motion ("jitter") as two-dimensional image sensors are exposed and read out line by line ("rolling shutter"). The results indicate that the Europa Imaging System (EIS) on NASA's Europa Clipper can likely meet its scientific goals requiring 0.1-pixel precision. We are therefore adapting the software used to demonstrate and test rolling shutter jitter correction to become part of the standard processing pipeline for EIS. The correction method will also apply to other rolling-shutter cameras, provided they have the operational flexibility to read out selected "check lines" at chosen times during the systematic readout of the frame area.
\end{abstract}

\section{BACKGROUND}

Remote imaging has been a key tool for planetary investigation since the earliest days of the space age, but the technology has evolved greatly. Each new type of sensor (film, vidicon, charge-coupled device or CCD, and active pixel sensor or APS) has conferred improved capabilities but created new processing challenges.

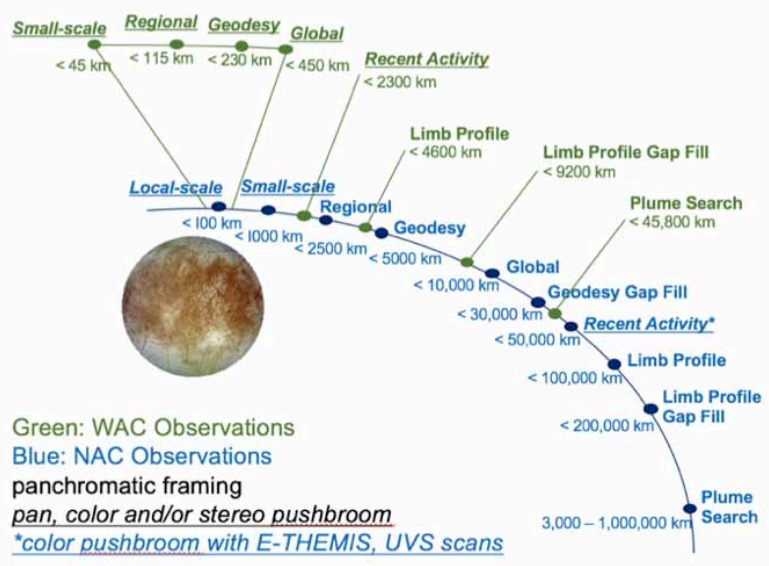

Figure 1. Typical sequence of EIS observations during a Europa flyby, including both pushbroom and framing images.

Our study is part of the design effort for EIS (Turtle et al. 2016), which consists of two cameras with different optics but identical APS detectors and readout electronics: the Wide Angle Camera (WAC) with $48^{\circ} \times 24^{\circ}$ field of view (FOV) and the Narrow Angle Camera (NAC) with $2.3^{\circ} \times 1.2^{\circ} \mathrm{FOV}$. Selection of an APS was motivated in part by its ability to read out individual detector lines in any order. This allows the EIS cameras to operate as both pushbroom and frame sensors, collecting multiple image sequences of varying coverage and resolution during a typical Europa flyby (Figure 1). In pushbroom mode, the WAC has multi-line stereo and color capabilities, similar to the High Resolution Stereo Camera on Mars Express (Neukum et al. 2004). The NAC has color but its FOV is too small for useful pushbroom stereo. Geometric distortion of pushbroommode images will be corrected by the same approach (Sutton et al. 2017) used for the Mars Reconnaissance Orbiter High Resolution Imaging System (HiRISE; McEwen et al. 2007) by using overlapping lines at different down-track positions. Matching of features in the overlap areas yields time differences of the camera pointing, from which the absolute pointing history (apart from an overall pointing bias) can be modeled. The main difference is that whereas the intervals between lines are set by the focal plane design for HiRISE, for EIS they can be chosen anywhere on the APS chip.

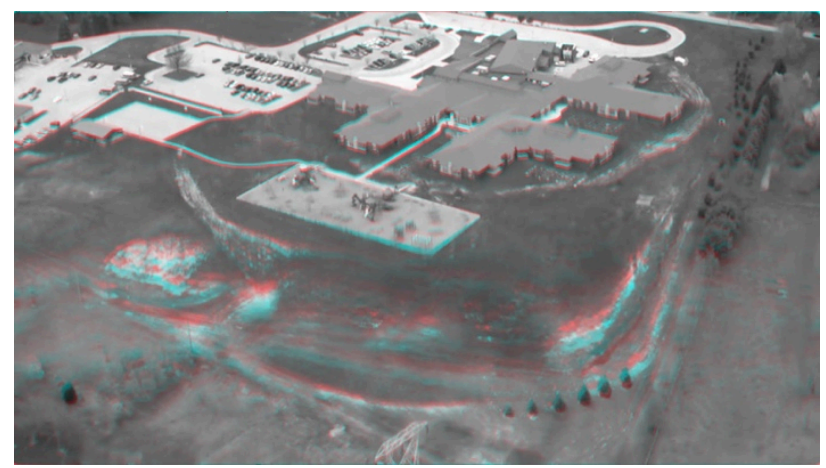

Figure 2. Example of extreme (tens of pixels) jitter distortion in images obtained from a helicopter with a handheld APS camera. Sequential frames from a video sequence have been combined in color to emphasize the changing geometric distortion. Video is online at https://www.youtube.com/watch? $\mathrm{v}=\mathrm{GdjP} 3$ aumV1E

The EIS cameras' large unfiltered area (1536 lines by 4032 samples) can be used to obtain two-dimensional "frame" images. Unfortunately, these lines must be read out sequentially over a period of $26 \mathrm{~ms}$, not simultaneously, so these images are also distorted by jitter. This phenomenon is now familiar from numerous aerial and extreme-sports videos taken with active pixel cameras (e.g., Figure 2). The distortion 
of EIS NAC images is expected to be smaller, but still scientifically significant. The specification for pointing stability of Clipper is $\pm 25 \mu \mathrm{Rad} / \mathrm{s}$, leading to an estimate of $4.5-15 \mu \mathrm{Rad}$ (a negligible fraction of a WAC pixel but 0.5-1.5 NAC pixels) during readout. The detailed spectrum of pointing variations is currently unknown but could extend up to $700 \mathrm{~Hz}$, so distortions could range from a slow drift to $\sim 18$ cycles across the image in any combination. Pixel-level distortion is acceptable for geologic studies but would interfere with geodetic and limbtopography observations with the NAC that require $\sim 0.1$ pixel precision. We therefore undertook to develop and demonstrate a technique for modeling and correcting rolling-shutter jitter distortion. If such correction cannot be done, the precision requirements for geodesy and limbs would have to be relaxed. A redesign of the electronics to permit simultaneous readout of all lines for true frame imaging has been judged prohibitively costly in terms of mass and complexity.

\section{APPROACH}

Our approach makes use of the flexibility of APS readout to obtain additional data from which the jitter can be estimated and corrected. The systematic (line by line) readout of the frame area is periodically interrupted to read some detector rows ("check locations") one or more additional times, resulting in "check lines." These are matched to the corresponding locations in the systematic readout and the resulting timedifferences of pointing are analyzed to produce a model of the pointing history (as in the pushbroom case, the absolute pointing bias cannot be determined from the differences). Because the expected motions are small, it is convenient to model them as translations in the image plane rather than as rotations of the camera.
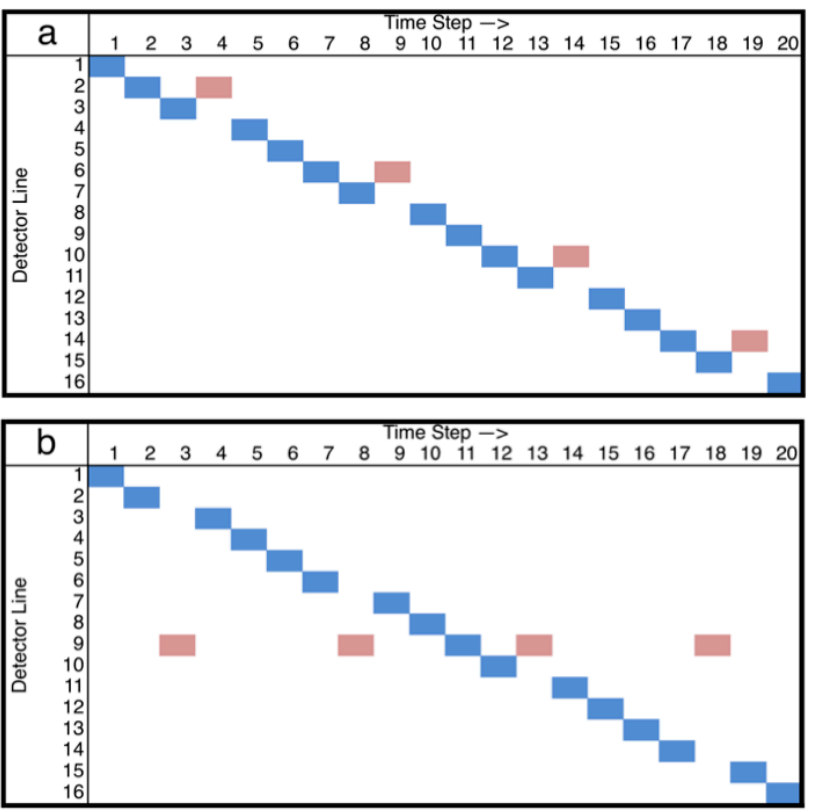

Figure 3. Schematic illustration of alternative strategies for reading check lines (red) by interrupting the systematic readout of the frame area (blue). (a) Check lines "shadow" a subset of the detector lines a short time after systematic readout. (b) A small number of check locations (for clarity only one is shown) are read out repeatedly.

The location and timing of the check lines has a strong impact on how the jitter is modeled. For example, "shadowing" the systematic readout (reading some lines a second time shortly after each is read systematically; Figure 3a) would produce a data set similar to that used for pushbroom mode, and a similar modeling approach (Sutton et al. 2017) could be used. If a single check location is used (not a disadvantage, since at any given instant all detector lines move together) the analysis is particularly simple because its pointing differences relative to the systematic read-out time are identical to the pointing history (apart from a constant offset). Although reading a small number of check locations (Figure 3b) makes the analysis slightly more complex (their delta-pointing histories must be adjusted to create a single pointing history), it has several advantages. First, each check location is read out less often, resulting in longer exposures and higher signal to noise ratio (SNR). Second, if the image texture at a single line is highly directional, the estimates of sample and line components of the jitter will be correlated. This is likely to be a severe problem for limb images, where the main "feature" is the limb itself, but could also be an issue for non-limb images because of the prevalence of parallel linear features on Europa. Checking several locations can break this correlation of the sample and line estimates.

\section{PROOF OF CONCEPT}

To evaluate the approach outlined above, we implemented software to simulate jittery images by resampling a "truth" image as well as to model and correct the distortions.

\subsection{Software}

Steps in the simulation and processing were implemented as individual applications that are compatible with, but not currently distributed as part of, the U. S. Geological Survey's Integrated Software for Imagers and Spectrometers, v.3.x (ISIS3; Sides et al. 2017). These steps, illustrated in Figure 4, are as follows.

- Create, from user input, a text file defining the "true" jitter to be applied to the image. An arbitrary number of harmonic terms with arbitrary frequencies and independent amplitudes and phases in sample and line can be specified.

- Create, from user input, a text file defining the schedule for reading out the systematic image lines and check lines, allowing any number of check locations and repeat readings.

- Resample the truth image according to the jitter and schedule definitions, placing the systematically read image in one file and the check lines in another.

- Match each check line to the corresponding neighborhood of detector lines in the systematic image, yielding a list of deltapointing values in samples and lines for known pairs of readout times. Normalized cross-correlation (NCC; Pratt 1991) is used to identify the best whole pixel alignment of systematic and check imagery, and the correlation is then interpolated in the neighborhood around this offset location to estimate the optimum to a fraction of a pixel. Additional enhancements to this procedure are described below.

- Fit a polynomial model of the pointing history (independently in sample and line directions) to the match data. Such polynomials are first fit separately for each check location, then their biases are adjusted to place them on a single curve and the model is re-estimated using all check lines. 
- Resample the distorted systematic image, using the modeled pointing history to place pixels at their undistorted locations, as described by the fitted model.

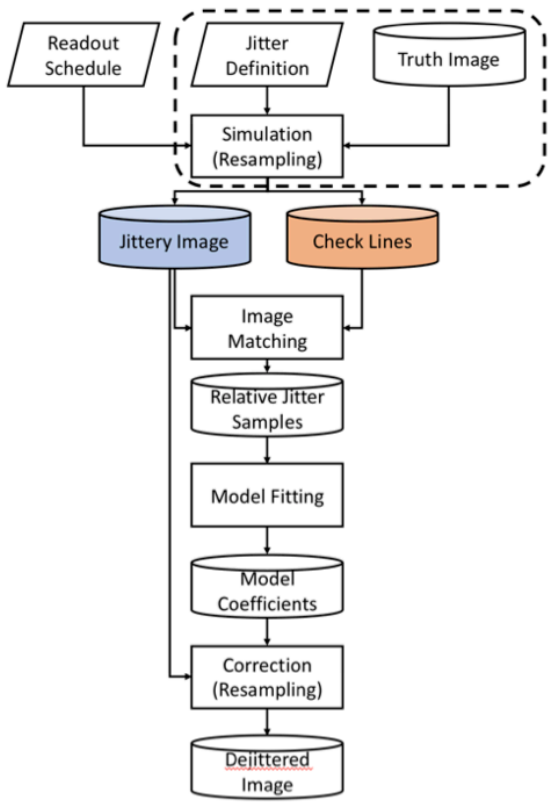

Figure 4. Workflow for simulating images with rolling-shutter jitter distortions (components outlined with a dashed line at upper right) and testing the ability to recover undistorted images. Systematically read main image and check lines are color-coded as in Fig. 3.

\subsection{Test Data}

A $210 \mathrm{~m} /$ pixel mosaic of Galileo images of Europa (Figure 5) was selected as the truth image. This is the same mosaic used in our simulations of the impact of illumination angles on EIS stereo (Kirk et al. 2016). To simulate a limb image, the portion of the mosaic outside a circular arc was set to zero.
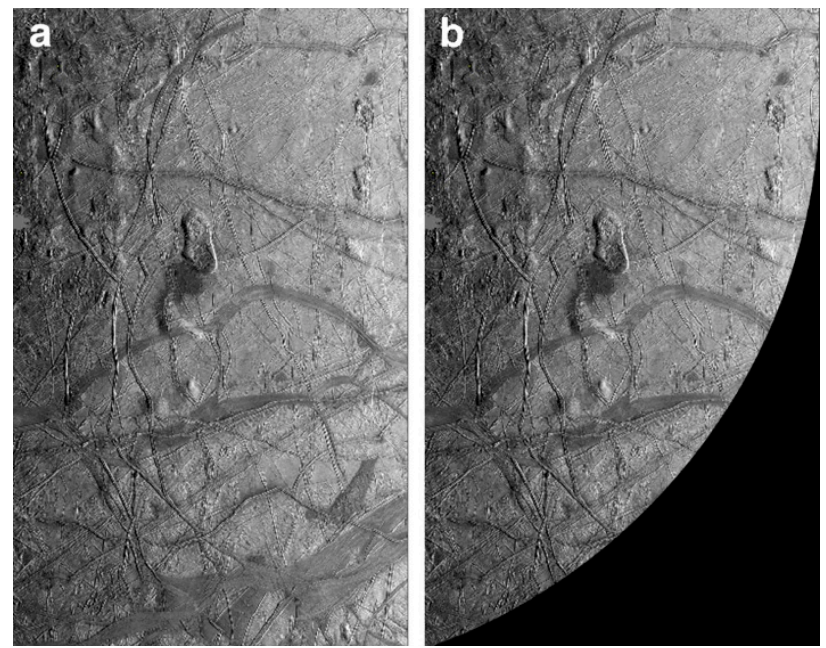

Figure 5. "Truth" image used for testing, a mosaic of six Galileo images at $210 \mathrm{~m} /$ pixel, covering $294 \times 472.5 \mathrm{~km}$ centered on Castalia Macula $\left(0.7^{\circ} \mathrm{N}, 225.3^{\circ} \mathrm{W}\right)$. (a) The mosaic does not include the limb of Europa. (b) Stenciling the mosaic with a circular mask simulates a limb view.

\section{RESULTS}

We selected three check locations well distributed across the image field, each checked 20 times. The jitter was approximately a full cycle over the duration of the readout, with arbitrary phases and different amplitudes (near but not exactly one pixel) in sample and line (Figure 6).

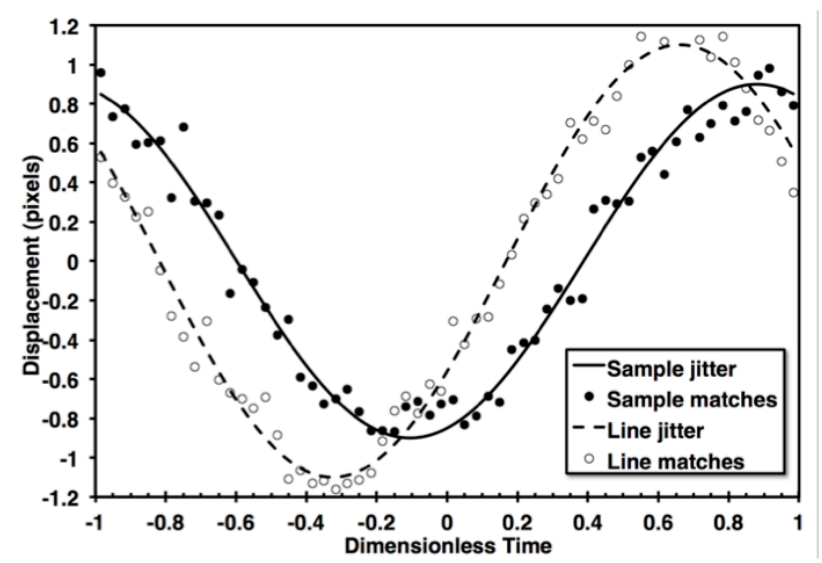

Figure 6. Jitter displacements used to resample the "truth" image for the tests described here and the displacements recovered by matching check lines to the image. Time has been scaled to run from -1 to 1 over the readout period.

The 0.5-pixel root-mean-square (RMS) difference between the true and matched displacements in our first tests was disappointing. Matching errors of 0.2 pixel are commonly achieved in stereo mapping (Kirk et al. 2016), and errors for matching two versions of the same image should be even smaller. Matches also appeared to cluster near a few preferred values, suggesting that "pixel locking" could be affecting the results. This is a well-known problem in which area-based subpixel matches tend to be biased toward whole-pixel offsets (Prasad et al. 1992) We therefore implemented two published strategies for mitigating pixel locking. The first simply involves enlarging the images by smooth interpolation (in our case by $4 \mathrm{x}$ ) so that locking occurs at a level inside the original pixels (Debela-Gilo amd Kääb 2011). The second averages the standard subpixel match location with one computed after interpolating one of the images to displace it by 0.5 sample and line. The "half-pixel locking" of the second estimate acts to cancel the pixel locking of the first (Shimizui and Okitomi 2005).

We eventually traced the poor early results to our failure to search a large enough range of offsets for the optimal displacement, causing the subpixel step to fail. With this problem corrected, even the basic matching strategy achieves the desired precision of $\sim 0.1$ pixel in sample and line individually (Table 1) but the anti-locking approaches yield even smaller RMS errors and are thus valuable. The larger errors for the limb image were unexpected and may result from the limb mask not being adequately anti-aliased. Note that these are errors between individual matches and the true displacements of the respective lines. Because the jitter model is fitted through many such estimates, its deviation from the true jitter is even smaller. For example, in the non-limb case with standard matching, the RMS error in individual matches is 0.109 sample but the fitted polynomial has a RMS error of only 0.035 sample. 


\begin{tabular}{|l|l|l|l|l|l|l|}
\hline & \multicolumn{3}{|c|}{ Non-Limb } & \multicolumn{3}{c|}{ Limb } \\
\hline Method & Sample & Line & RSS & Sample & Line & RSS \\
\hline Standard & 0.109 & 0.104 & 0.148 & 0.141 & 0.158 & 0.212 \\
\hline Enlarged & 0.041 & 0.055 & 0.069 & 0.074 & 0.075 & 0.105 \\
\hline $1 / 2$ Pixel & 0.081 & 0.077 & 0.112 & 0.079 & 0.114 & 0.139 \\
\hline
\end{tabular}

Table 1. RMS matching errors in pixels. RSS = root sum square of sample and line errors.

We have not yet performed a full simulation (resampling the "truth" image and performing image matching) for jitter with higher frequencies, but a simplified calculation indicates that such jitter can be recovered with a modest number of noisy measurements. In this calculation we defined a jitter pattern with about 9 cycles (roughly half the highest frequency expected). We took 30 uniformly spaced samples of this pattern and perturbed them with pseudo-random noise with a standard deviation of 0.1 pixel. Finally, we fitted a model jitter function through these points. Because fitting polynomials becomes numerically unstable as their order increases, we used a sinusoid with adjustable frequency, phase, and amplitude as the fitting function. This model was found to fit the true jitter with an RMS error of only 0.03 pixel. This is consistent with the full simulation results for lower frequency jitter.

\section{REMAINING WORK}

Considerable work remains to incorporate rolling-shutter jitter correction into the EIS uplink and downlink pipelines and test it with real data. The components outlined with a dashed line in Fig. 4 will be replaced by the real camera, target, and spacecraft dynamics so are needed only for simulations. The program to schedule check lines will be used and the flight operations team will use our schedule files to build commands to the camera. Onboard the spacecraft, the systematically read frame image and the ensemble of check lines will be recorded as two separate files. The former can be compressed normally but the check lines require minimal (ideally, lossless) compression to avoid compromising the subtle information about sub-pixel to pixel level offsets. Label information for each file will include the time at which each row was exposed and read out.

We are presently combining our line-matching and jitter-fitting programs into a single ISIS3 application that will be used to model the jitter in flight images. The jitter correction step will be incorporated into the camera model for EIS, which will correct jitter distortions immediately before correcting optical distortion in projecting from image to ground space, and add back the distortions immediately after adding optical distortion when projecting from ground to image. The correction will be used "on the fly" in geodetic control of images by bundle adjustment and projection to make image mosaics. It can also be used to project images to the ground and back into a distortion-free "ideal" version of EIS, yielding an undistorted but otherwise geometrically "raw" image that can be used by other software, e.g., for stereo mapping, that does not perform jitter correction on the fly (cf. Kirk et al. 2008).

Because the frequency spectrum of jitter on Europa Clipper cannot be predicted precisely, experimentation will be required in the commissioning and early operations phases of the mission. We will collect images with a generous number of check lines and with various onboard sources of vibration turned off singly and in combinations to the extent possible. This will enable us to determine which instruments, etc., need to be inactive during NAC imaging and the minimum number of check lines needed for jitter correction.
Depending on the maximum frequency causing significant distortion, it may also become necessary to extend the jitter model that is fitted. As already noted, polynomial fits become unstable as the order increases. Experiments with modeling pushbroom jitter as part of the bundle adjustment process indicate that the maximum useful order is about 20, which suffices to model jitter with no more than about 7 cycles over the exposure period. This corresponds to about $270 \mathrm{~Hz}$ for EIS, so to model jitter that includes higher frequencies we will have to fit a different class of functions such as splines.

\section{CONCLUSION}

Matching a small number of check lines obtained throughout the systematic readout provides a viable basis for correcting geometric distortions caused by jitter. The achieved accuracy meets the requirement for EIS geodetic and limb topography imaging. We see no reason the technique would not also apply to any rolling-shutter sensor with similar flexibility in scheduling the readout.

\section{REFERENCES}

Debela-Gilo, M., and Kääb, A., 2011. Sub-pixel precision image matching for measuring surface displacements on mass movements using normalized cross-correlation. Remote Sensing of Environment, 115, pp. 130-142.

Kirk, R.L., Howington-Kraus, E., Hare, T.M., and Jorda, L., 2016. The Effect of Incidence Angle on Stereo DTM Quality: Simulations in Support of Europa Exploration. ISPRS Annals of Photogrammmetry Remote Sensing and Spatial Information Sciences, III-4, pp. 103-110, doi:10.5194/isprs-annals-III-4-1032016.

Kirk, R.L., Howington-Kraus, E., Rosiek, M.R., Anderson, J.A., Archinal, B.A., Becker, K.J., Cook, D.A., Galuszka, D.M., Geissler, P.E., Hare, T.M., Holmberg, I.M., Keszthelyi, L.P., Redding, B.L., Delamere, A.W., Gallagher, D., Chapel, J.D., Eliason, E.M., King, R., McEwen, A.S., and the HiRISE Team, 2008. Ultrahigh resolution topographic mapping of Mars with MRO HiRISE stereo images: Meter-scale slopes of candidate Phoenix landing sites. Journal of Geophysical Research, 113, E00A24, doi:10.1029/2007JE003000.

McEwen, A.S., Eliason, E.M., Bergstrom, J.W., Bridges, N.T., Hansen, C.J., Delamere, W.A., Grant, J.A., Gulick, V.C., Herkenhoff, K.E., Keszthelyi, L., Kirk, R.L., Mellon, M.T., Squyres, S.W., Thomas, N., and Weitz, C.M., 2007. Mars Reconnaissance Orbiter's High Resolution Imaging Science Experiment (HiRISE). J. Geophys. Res., 112, E05S02, doi:10.1029/2005JE002605.

Neukum, G., Jaumann, R., and the HRSC Co-Investigator Team, 2004. HRSC: The High Resolution Stereo Camera of Mars Express. ESA Special Publication. SP-1240.

Prasad, A., Adrian, R., Landreth, C., \& Offutt, P., 1992. Effect of resolution on the speed and accuracy of particle image velocimetry interrogation. Experiments in Fluids, 13, pp. 105116.

Pratt, W. K., 1991. Digital Image Processing, $2^{\text {nd }}$ Ed., Wiley, New York.

Shimizu, M., and Okutomi, M., 2005. Sub-Pixel Estimation Error Cancellation on Area-Based Matching. International Journal of Computer Vision, 63, pp. 207-224. 
Sides, S.C., Becker, T.L., Becker, K.J., Edmundson, K.L., Backer, J.W., Wilson, T.J., Weller, L.A., Humphrey, I.R., Berry, K.L., Shepherd, M.R., Hahn, M.A., Rose, C.C., Rodriguez, K., Paquette, A.S., Mapel, J.A., Shinaman, J.R., Richie, J.O., 2017. The USGS Integrated Software for Imagers and Spectrometers (ISIS 3) Instrument Support, New Capabilities, and Releases. Lunar and Planetary Science, XLVIII, 2739.

Sutton, S., Boyd, A., McEwen, A., Fennema, A., Heyd, R., Mirchandini, S., Kirk, R., Cook, D., and Backer, J., 2017. Correcting Spacecraft Jitter in HiRISE Images. International Archives of Photogrammetry, Remote Sensing, and Spatial
Information Sciences, XLII-3-W1, pp. 49-53, doi:10.5194/isprsarchives-XLII-3-W1-49-2017.

Turtle, E.P., McEwen, A.S., Collins, G.C., Fletcher, L., Hansen, C.J., Hayes, A.G., Hurford, T.A., Kirk, R.L., Barr Minar, A.C., Nimmo, F., Patterson, G.W., Quick, L.C., Soderblom, J.M., Thomas, N., Ernst, C.M., 2016. The Europa Imaging System (EIS): High-Resolution Imaging and Topography to Investigate Europa's Geology, Ice Shell, and Potential for Current Activity. Lunar and Planetary Science, XLVII, 1626. 